\title{
O papel do enfermeiro na humanização do parto normal
}

\author{
The role of nurses in humanizing normal children
}

El papel de las enfermeras en la humanización de los niños normales

\begin{abstract}
Adrian Thaís Cardoso Santos Gomes da Silva ${ }^{1 *}$, Rayanne Lúcia de Oliveira Campos ${ }^{1}$, Niedja Carla Dias de Lira e Silva ${ }^{2}$, Luan Naís de Souza ${ }^{3}$, Manoela Rodrigues de Santana ${ }^{4}$, Ada Evellyn Galdino da Silva ${ }^{5}$, Luany Abade Café ${ }^{1}$, Edivania Cristina da Silva ${ }^{6}$, Paloma Maria Oliveira de Almeida ${ }^{3}$, Amanda Domingos da Silva ${ }^{7}$.
\end{abstract}

\section{RESUMO}

Objetivo: Este artigo buscou discutir sobre a humanização do parto e seus métodos de funcionamento, tendo como objetivo descrever o papel do enfermeiro na assistência humanizada prestada durante o trabalho de parto normal. Revisão bibliográfica: Diante do exposto, observou-se que os enfermeiros buscam orientar as parturientes acerca da evolução do parto e suas respectivas fases, e assim, proporcionam maior conforto físico e emocional a elas. Além de encorajá-la a adotar posições que favoreçam a descida do feto, bem como, contribuem na redução de intervenções desnecessárias, como a episiotomia, e as orientam sobre os métodos não farmacológicos e seus benefícios. Considerações finais: De fato, a equipe de enfermagem como um todo contribui positivamente nesta fase por meio da prática da humanização, fazendo-se necessário que os profissionais envolvidos mantenham suas condutas sempre atualizadas e baseadas em evidências científicas a fim de manter as boas práticas de assistência baseadas nos princípios da humanização.

Palavras-chave: Parto humanizado, Humanização da assistência, Papel do profissional de enfermagem.

\begin{abstract}
Objective: This article sought to discuss the humanization of childbirth and its methods of operation, with the objective of describing the role of the nurse in the humanized care provided during normal labor. Bibliographic review: In view of the above, it was observed that the nurses seek to guide the women on the evolution of childbirth and its respective phases, and thus provide them with greater physical and emotional comfort. Besides encouraging them to adopt positions that favor the descent of the fetus, they also contribute in the reduction of unnecessary interventions, such as episiotomy, and guide them on non-pharmacological methods and their benefits. Final considerations: It is concluded that, in fact, the nursing team as a whole contributes positively in this phase through the practice of humanization, making it necessary that the professionals involved keep their conduct always updated and based on scientific evidence in order to maintain good practices of assistance based on the principles of humanization.
\end{abstract}

Keywords: Humanizing delivery, Humanization of assistance, Nurse's role.

\section{RESUMEN}

Objetivo: Este artículo pretendía discutir la humanización del parto y sus métodos de operación, con el objetivo de describir el papel de la enfermera en los cuidados humanizados que se proporcionan durante el parto normal. Revisión bibliográfica: En vista de lo anterior, se observó que las enfermeras tratan de orientar a las mujeres sobre la evolución del parto y sus respectivas fases, y así proporcionarles una mayor comodidad física y emocional. Además de alentarlos a adoptar posiciones que favorezcan el descenso del feto, así como

1 Universidade Federal de Pernambuco, Recife - PE. *E-mail: adrianthais@hotmail.com

2 Centro Universitário São Miguel (Uni São Miguel), Recife - PE.

${ }^{3}$ Faculdade Pernambucana de Saúde, Recife - PE.

${ }^{4}$ Universidade Católica de Pernambuco, Recife - PE.

${ }^{5}$ Centro Universitário Estácio de Recife, Recife - PE.

${ }^{6}$ Centro Universitário dos Guararapes (UNIFG), Jaboatão dos Guararapes - PE.

${ }^{7}$ Centro de Ensino e Pesquisa em Emergências Médicas, Recife - PE. 
contribuir a reducir las intervenciones innecesarias, como la episiotomía, y orientarlos sobre los métodos no farmacológicos y sus beneficios. Consideraciones finales: Se concluye que, de hecho, el equipo de enfermería en su conjunto contribuye positivamente en esta fase mediante la práctica de la humanización, lo que hace necesario que los profesionales implicados mantengan su conducta siempre actualizada y basada en la evidencia científica para mantener unas buenas prácticas asistenciales basadas en los principios de la humanización.

Palabras clave: Parto humanizado, Humanización de la atención, Rol de la enfermera.

\section{INTRODUÇÃO}

O parto vaginal historicamente era tratado como um processo natural e fisiológico, o qual era vivenciado pela mulher em seu domicílio, com auxílio de parteiras ou de mulheres que possuíam conhecimento empírico sobre o processo da gestação e parto. Contudo, a partir do século $\mathrm{XX}$, iniciou-se a substituição do parto domiciliar pelo parto hospitalar, onde foram incluídos rotinas e procedimentos médicos, como a episiotomia e o uso de fórceps rotineiramente (MOURA FMJSP, et al., 2007).

A hospitalização do parto trouxe consigo condutas e práticas intervencionistas, que foram tirando da mulher a autonomia que ela possuía durante todo o processo. Assim, o parto tornou-se um momento de procedimentos desnecessários, internamento precoce, traumas, sofrimento físico, psíquico e moral (SANCHES METL, et al., 2019).

No Brasil, a Organização Mundial de Saúde (OMS) considera epidêmica a elevação das taxas de cesárea, com valores que superam os 15\% preconizados. Os Indicadores e Dados Básicos para a Saúde do Brasil fornecem, em seus índices de cobertura, a proporção de partos cesáreos, cujos valores alcançaram o patamar de 52,34\%, no ano de 2010 (VELHO MB, et al., 2014).

No cotidiano dos serviços de saúde, observa-se que as usuárias da rede pública de saúde não costumam participar da decisão pelo tipo de parto, sendo essa definida por conduta médica. As usuárias da rede privada também costumam se submeter à cesariana eletiva, em função da maior comodidade, tanto para a mulher, quanto para o(a) profissional médico(a). Já no ano de 2018, a proporção de partos em hospitais segundo esfera administrativa foi: Pública com, $61,7 \%$ vaginais - 38,3\% cesáreos; e Não pública representado por $34 \%$ vaginais $-65,9 \%$ cesáreos (SANTOS GO, et al., 2018).

A humanização envolve práticas e condutas desenvolvidas a partir da criação do Programa de Humanização do Pré-natal e Nascimento (PHPN), que traz recomendações de práticas e abordagens terapêuticas baseadas em evidências científicas, dentre os quais temos a presença de um acompanhante de livre escolha da mulher (POSSATI AB, et al., 2017). As boas práticas de atenção ao parto e nascimento são ações benéficas que visam diminuir a morbimortalidade materna e neonatal. Quando essas práticas deixam de serem realizadas coloca em risco a saúde da mulher e do neonatal (ALVES TCM, et al., 2019).

Atualmente, profissionais de saúde, principalmente o enfermeiro, vem tentando desmistificar o antigo modelo assistencial ao parto, tornando-o mais humanizado. $O$ cuidado humanizado tem como premissa, 0 resgate do parto natural, onde a mulher é protagonista. Para tanto, essa experiência necessita de um novo olhar, onde a mulher consiga ser ouvida, acolhida, orientada e tenha as suas vontades respeitadas (MEDEIROS RMK, et al., 2016).

A enfermagem tem conquistado espaço nas políticas públicas devido ao olhar qualificado durante o processo do parto, adotando medidas para que esse processo ocorra com o mínimo de intervenções e construindo vínculo com a mulher e a família desde as consultas de pré-natal até o puerpério (ALVES TCM, et al., 2019). A prática assistencial das enfermeiras obstétricas é voltada para a valorização da mulher, fortalecendo-a no processo de parir, acolhendo, respeitando-a em seu tempo, propiciando cuidados para o alívio da dor e condução do trabalho de parto, estimulando-a a sua execução como: exercícios, massagens, banhos, deambulação, e adoção de posições mais verticalizadas (DUARTE MR, et al., 2020).

Nesse sentido, o presente estudo objetivou descrever o papel do enfermeiro na humanização do parto normal, assim como, suas contribuições para com as mulheres, RNs e familiares, e destacar seus benefícios 
e resultados positivos. Além de contribuir e proporcionar tanto para os profissionais de enfermagem quanto para a comunidade acadêmica, maiores conhecimentos relacionados à temática abordada. Com o intuito de proporcionar melhorias na assistência às parturientes através do olhar holístico e uma assistência humanizada.

\section{REVISÃO BIBLIOGRÁFICA}

\section{Humanização no contexto da saúde}

O Hospital Mont Sinai, em Boston (EUA), em 1972 emitiu a declaração dos direitos dos pacientes que foi reconhecida pela literatura, pois desde o início dos anos 1970 passou-se a discutir o tema humanização refletindo sobre práticas humanizadoras em saúde e direitos do paciente. A partir do simpósio americano cujo título Humanizing Health Care (Humanizando o Cuidado em Saúde), no ano de 1972, em São Francisco, foi considerado o marco do movimento de humanização em saúde. A discussão sobre humanização em saúde e a Declaração da Conferência Internacional Sobre Cuidados Primários em Saúde, realizada em Alma-Ata na capital do Cazaquistão, em 1978 "reafirmou que a saúde é um estado de Bem-estar completo, físico, mental e social e não somente a ausência de doença ou enfermidade" (MEDEIROS LMOP e BATISTA SHSS, 2016).

O Programa de Humanização do Pré-natal e Nascimento (PHPN) foi criado em 2000. Segundo o mesmo, a humanização abrange o acolhimento digno à tríade composta por: mulher-bebê-família, mediante ações éticas e solidárias. Tendo como objetivo qualificar a assistência ao pré-natal, garantindo também seu acesso e cobertura completa, tal como aprimorar a atenção aos processos materno-infantil (POSSATI AB, et al., 2017).

O atendimento humanizado na saúde tem uma ligação direta com as condições de trabalho e salário do profissional de saúde, ter uma quantidade adequada de profissionais trabalhando, uma relação ética entre a ação humanizada e a ética no trabalho, colocar a ação humanizadora como base da ética profissional, está preparado para as relações humanas, conhecimento técnico e científico, satisfação no trabalho realizado, autoconhecimento, pois conhecendo a si mesmo ajuda a tomar ciência das próprias limitações e a melhor maneira de ultrapassá-las, ter atividades educativas e educação continuada, assim descobrindo suas potencialidades e reforçando o conhecimento teórico, para melhor atender o paciente de forma humanizada (MEDEIROS LMOP e BATISTA SHSS, 2016).

De acordo com a Política de Humanização da Assistência à Saúde (PHAS), criada em 2003, ao qual estabelece diretrizes que contemplam os projetos de caráter humanizado, desenvolvidos em várias instituições de saúde, possui como objetivo respeitar as individualidades de cada instituição e possibilitar a cooperação entre elas em busca da troca de vivências que possibilitem aprimorar a qualificação do serviço público de saúde, permitindo a continuidade de espaços de comunicação e divulgação, que possibilitem e estimulem a livre expressão, o diálogo, o respeito e a solidariedade (MORAIS T, 2016).

A palavra humanização também pode ser definida como ato de tornar humano, civiliza-se, humanizar-se. Humanização na atenção à saúde passa a compreender cada pessoa do seu jeito, baseado em um relacionamento construído de respeito, empatia e carinho, entre o paciente e o profissional de saúde. De modo que esse profissional possua um novo olhar e percepção para com o seu cliente. Criando um vínculo por meio da escuta ativa e orientação dentro do acolhimento (POSSATI AB, et al., 2017; PAGANINI AA, et al., 2018).

A humanização acontece com transformações políticas, administrativas e subjetivas, uma mudança no modo de ver o paciente, deixando de ser objeto passivo e passando para objeto ativo, ter diálogo passa a ser um ponto positivo, o conhecer o outro sabendo ouvir e falar, compreendendo-o com ser humano. O humanista dá valor aos seres, a vida e a dignidade humana, com visão consciente da liberdade do indivíduo, sua razão, suas oportunidades e seus direitos. Na formação profissional é preciso ter uma visão de política transversal, ultrapassando fronteiras, podem ser muitas vezes rígidas dos diferentes conhecimentos na promoção a saúde, cujo trabalho será em longo prazo, dinâmico e está intimamente relacionado no que se envolve, precisando muitas vezes esconder suas emoções, para não ser pontuado como frágil ou sensível e não estar apto ao trabalho (MEDEIROS LMOP e BATISTA SHSS, 2016) 
No contexto do parto, o conceito de humanização se baseia em atitudes, práticas e condutas acolhedoras pautadas em conhecimentos metódicos no desenvolvimento saudável dos processos de parto e nascimento, de acordo com os desejos da mulher e suas particularidades. O momento do parto de fato é único, primípara ou multípara, cada parto a mulher terá uma vivência divergente e singular. O papel da humanização no parto se baseia em possibilitar que esse momento seja único e satisfatório tanto mulheres quanto para o recémnascido (RN) (POSSATI AB, et al., 2017).

\section{Parto Humanizado}

Conforme a Organização Mundial de Saúde (OMS) (2018), o parto está relacionado diretamente a uma vivência compartilhada entre a mulher e o bebê, possuindo caráter fisiológico e natural, podendo ocorrer sem quaisquer complicações (OMS, 2018). Com o intuito de garantir qualidade na assistência prestada à mulher em período gravídico, o Ministério da saúde (MS) desde a década de 80 vem desenvolvendo programas para humanização e criação de centros de partos naturais e o protagonismo da mulher nesse momento.

Garantindo assistência materno infantil e acesso ao pré-natal, assegurando-lhe uma assistência digna, com as informações necessárias para que possa escolher o local do parto, o tipo de parto, a assistência, quem irá lhe acompanhar, e, entre outras respeitando a participação de sua família. Essas medidas visam a humanização da assistência prestada à mulher e a redução de coeficientes da mortalidade materna e neonatal (SILVA DC, et al., 2015).

O parto é uma prática social vivido por mulheres e também seus familiares. Inicialmente era realizado por parteiras, nos domicílios. Com a criação dos hospitais a ideia do parto passou a sofrer algumas mudanças, de modo que deixou de ser um evento mais pessoal e particular passando a se tornar um ato médico. Diante disso, veio ocorrendo uma diminuição no protagonismo da mulher, passando esse papel para o médico, já que o mesmo que tomará partida sobre quais decisões serão mais cabíveis. Dessa forma, os partos cesarianos foram crescendo no universo da obstetrícia de forma a se tornarem primeira opção, muitas vezes sem nenhuma necessidade (TOSTES NA e SEIDL EMF, 2016).

A humanização do parto possibilita que a parturiente desfrute de sua autonomia e poder de escolha, engrandecendo sua autoconfiança e sentimento de controle diante desta situação. O empoderamento traz uma consciência e reduz os índices de intervenções cesarianas. Dessa maneira, proporcionando a redução da morbimortalidade materna e perinatal, e assim, caminhando para uma assistência mais humanizada (JÚNIOR S, et al., 2019).

O empoderamento é a consciência coletiva, expressada através de ações para fortalecer a figura feminina possibilitando o aumento da autonomia, liberdade, o pensamento reflexivo e crítico, dando a gestante as condições necessárias para entender as mudanças ocorridas na saúde e a escolha do seu acompanhante que é preconizada pela Lei Federal no 11.108, de 07 de abril de 2005, onde determina que os serviços prestados pelo Sistema Único de Saúde (SUS), em todo o período do trabalho de parto, parto e pós parto, são obrigados a permitir o direito ao acompanhante. Determinando que a indicação do acompanhante será exclusivamente feita pela gestante, a pessoa de sua escolha (MARINHO PAS e GONÇALVES HSG, 2016; MINISTÉRIO DA SAÚDE, 2005).

A atuação da humanização trás o maior conforto emocional por meio de boas práticas na assistência prestada, tranquilizando a parturiente, promovendo o restabelecimento mais rápido e criando uma experiência positiva na hora do parto. Em suas práticas estão incluídas, por exemplo, massagens, meios de relaxamento, exercícios respiratórios e a bola terapêutica, que vem proporcionando um parto mais tranquilo, reduzindo até o tempo de sua duração. Quanto a fase do pós-parto, essa assistência mais humanizada se dá a educação permanente que é oferecida às puérperas através do banco de leite, onde as mesmas serão instruídas sobre a prática da amamentação e seus benefícios (GUIDA NFB, et al., 2016).

\section{Papel do enfermeiro na humanização do parto normal}

Ao longo dos anos no Brasil o conceito do que se entende por parto passou por modificações de forma progressiva, dado que antes o parto era realizado a nível domiciliar com auxílio de parteiras e familiares, e atualmente é mais corriqueiro que ocorra em ambiente hospitalar, assim como é considerado um 
procedimento cirúrgico realizado por médicos, que refutam a ação das parteiras (MALHEIROS PA, et al., 2012).

Porém, essa história está sendo modificada, pois de acordo com a portaria $n^{\circ}$ 2.815/98 e 169/98 o parto normal sem distócia deve ser realizado pelo enfermeiro obstetra. Visto que a atuação dos profissionais de enfermagem na assistência obstétrica humanizada está quebrando arquétipos, agregando novos conhecimentos técnicos científicos e transformando o modelo biomédico obstétrico (SILVA AF et al., 2018).

Compreende-se por parto sem distorcia aquele que acontece de forma natural. As distorcia são circunstâncias adversas que desfazem o equilíbrio, prejudicando o processo natural do parto e requerendo cuidados mais complexos. Apesar de toda fragilidade, observa-se que o enfermeiro presta uma assistência humanizada mesmo que paulatinamente (BRASIL, 2017).

A Organização Mundial de Saúde e o Ministério da saúde têm intenção de incluir medidas que possam mudar a assistência ao parto, abarcando o resgate do parto natural a partir do apoio e atuação da enfermagem obstetra na assistência à gestação e parto (OMS, 2018).

Dessa forma, o enfermeiro atua no alívio da dor a parturiente por meio de em uma assistência mais humanizada e não farmacológica, tornando esse momento especial e com mais conforto. Existem métodos não farmacológicos comprovadamente benéficos na assistência ao parto normal, que trazem além do conforto e bem-estar, um ambiente acolhedor e a possibilidade de maior apoio por parte de toda a equipe multidisciplinar. As boas práticas obstétricas durante o trabalho de parto possibilitam a utilização de recursos não medicamentosos, são exemplos dos métodos não farmacológicos de alívio da dor, aromaterapia, musicoterapia, massagens, doula e/ou acompanhante de escolha da mulher e técnicas de respiração. (PEREIRA TCB, 2016). Os métodos não farmacológicos tendem a valorizar o parto normal e à aplicação correta da assistência à parturiente de forma humanizada, buscando o alívio à dor e diminuir os efeitos colaterais (BRASIL, 2016).

Em 1996 a Organização Mundial de Saúde publicou o guia: "Assistência ao parto normal: um guia prático", oferecendo melhor qualidade na assistência a mulher e ao recém-nascido, onde se encontram condutas comprovadamente benéficas na assistência do parto normal, tencionando para que as boas práticas obstétricas fossem aplicadas em todos os países, oferecendo melhor qualidade na assistência a mulher e ao recém-nascido (OMS, 2000).

Neste contexto, a OMS preconiza que o parto aconteça o mais natural possível, evitando induções e intervenções desnecessárias, proporcionando a parturiente atendimento humanizado. Fazem parte das boas práticas na hora do parto: respeito e privacidade, liberdade de posição, dieta livre, exercícios que aceleram o trabalho de parto, métodos não farmacológicos para o alívio da dor, doula e/ou acompanhante nascido de sua escolha (BRASIL, 2017).

Os profissionais da saúde passaram a entender a relação do trabalho de parto dentro do contexto da humanização, por meio dos resultados obtidos através da influência positiva do acolhimento bem qualificado em associado às boas práticas assistenciais durante o processo de parturição. Além disso, reconheceram a necessidade de diminuir as práticas intervencionistas, bem como reconhecer a importância do direito a um acompanhante como uma ação positiva para a mulher. Todas essas ações com a finalidade de promover o bem-estar para mulher, e assim trazer à tona seu protagonismo no decorrer do trabalho de parto (LIMA LPM, et al., 2015).

Nesse contexto, o mesmo desenvolve um papel essencial na construção da humanização, tanto para as parturientes quanto para a equipe profissional, que em sua maioria apresenta um perfil de imposição sobre a condução do trabalho de parto. O enfermeiro obstetra e todas as suas práticas em sala de parto estão embasados pela resolução do Cofen $n^{\circ} 0516 / 2016$. O enfermeiro presta uma assistência mais humanizada a mulher durante o período de parturição, compreendendo não só a parturiente como os seus familiares, respeitando suas individualidades dando atenção às necessidades específicas, ultrapassando as questões naturais, envolvendo circunstâncias sociais, éticas, educacionais, e psíquicas presente em relacionamentos humanos (CAUS ECM, et al., 2017). 
O enfermeiro é responsável por orientar a gestante e a sua família desde a solicitação de exames específicos, de acordo com o protocolo liberado pelo MS, como na realização das consultas pré-natal de baixo risco e alto risco, cabendo ao enfermeiro realizar o encaminhamento, até na assistência intraparto e pósparto. No que se diz respeito à atenção básica, ele atua em grupos de educação continuada dentro das unidades de saúde da família (USF) desmistificando o parto natural e realizando anotações na caderneta da gestante. Desse modo, é criado um vínculo entre o enfermeiro, a gestante e seus familiares através do acolhimento ativo (LIMA LPM, et al., 2015).

A enfermagem vem se tornando referência de atenção a assistência da mulher, pois vem construindo com suas técnicas baseadas em instrumentos científicos e humanísticos, garantindo a mulher uma atenção integral e respeitosa em todo o ciclo-gravídico. Tal que, enfermagem obstétrica deve assistir ao parto, respeitando o processo fisiológico. Durante esse momento a assistência tem que possuir delicadeza, maior disponibilidade de tempo, prestando assim o cuidado necessário a parturiente estabelecendo uma relação de empatia, sem pressa, a mesma deve ser contínua para que a mulher se sinta segura com todos os profissionais que fazem parte da equipe (CAUS ECM, et al., 2017).

Ao avaliar a competência da enfermagem obstétrica no âmbito hospitalar observa-se um aspecto multidisciplinar, onde o profissional consegue atuar baseado em técnicas humanizadas, bem como 0 conhecimento intuitivo, pois o parto natural é imprevisível e o enfermeiro consegue adquirir esta habilidade que corrobora com o saber-fazer de qualidade. A atuação da equipe de enfermagem obstétrica na assistência ao parto de risco habitual vem sendo uma medida capaz de reduzir consideravelmente as intervenções desnecessárias durante o trabalho de parto e parto garantindo um cuidado integral à parturiente e a família (ALVES TCM, 2019).

Perante esse pressuposto, deve-se proporcionar um parto com experiências positivas para as gestantes e seus familiares. Logo, a assistência prestada com métodos não farmacológicos provê a futura mãe, meios para a diminuição das dores, ocasionando o relaxamento corporal durante o trabalho de parto, sem que haja o risco de uso indesejado dos analgésicos sintéticos (ALVES TCM, et al., 2015).

\section{CONSIDERAÇÕES FINAIS}

Evidencia-se o quanto a assistência humanizada priorizando o alívio da dor, conforto e maior protagonismo da mulher, é efetiva, bem como, evidenciada cientificamente e recomendada. Isto posto, o parto vaginal ou parto humanizado que representa uma conquista histórica como um dos direitos conquistados pelas gestantes, e também como estratégia de trazê-la para dentro desse momento único e especial, podendo ser acompanhada por alguém de sua escolha. Com isso, a enfermagem vem mostrando seu papel de extrema importância para o sucesso dessa humanização que representa a finalização de um ciclo longo e que muitas vezes tende a deixar traumas na vida da gestante, se realizado de modo errôneo. O vínculo e confiança devem ser estabelecidos entre o profissional e a gestante desde a atenção básica até a atenção hospitalar. Partindo do pressuposto, a enfermagem deve manter-se sempre atualizada, utilizando das evidências científicas como fonte para realizar uma assistência de excelência.

\section{REFERÊNCIAS}

1. ALVES TCM, et al. Contribuições da enfermagem obstétrica para as boas práticas no trabalho de parto e parto vaginal. Enfermagem em Foco, 2019;10(4): 54-60.

2. ANTUNES TFF. Medidas não invasivas para o alívio da dor durante o trabalho de parto. Trabalho de Conclusão de Curso, Universidade de Cuiabá, 2018;15.

3. BRANDT GP, et al. O papel da enfermagem no parto humanizado. Rev. Gestão e Saúde, 2016; 15(1): 34-38.

4. BRASIL, Ministério da saúde. Lei oํ 11.108 de 07 de abril de 2005. Dispõe sobre a presença de acompanhante durante o trabalho de parto, parto e pós-parto imediato, no âmbito do sistema único de saúde - SUS. 2005.

5. CAUS ECM, et al. O processos de parto assistido por a enfermeira obstétrica no contexto hospitalar: significados para as parturientes. Esc Anna Nery, 2017; 16(1):34-40

6. DUARTE MR, et al. Percepção das enfermeiras obstétricas na assistência ao parto: resgate da autonomia e empoderamento da mulher. 2020; 12:903-908.

7. GUIDA NFB, et al. O ambiente de relaxamento para humanização do cuidado ao parto hospitalar. Rev. Mineira de Saúde, 2016;17(3): 524-530. 
8. JÚNIOR S, et al. Desafios da enfermagem frente ao parto humanizado: uma revisão integrativa. Rev Multidiscip em Saúde, 2019;1(1): 36-43.

9. LIMA LPM, et al. O papel do enfermeiro durante a consulta de pré-natal à gestante usuária de drogas. Revista espaço para a saúde, 2015;16(3): 39-46.

10. MALHEIROS PA, et al. Parto e Nascimento: saberes e práticas humanizadas. Texto Contexto Enferm, 2012 Abr-Jun; 21(2): 329-37.

11. MARINHO PAS, GONÇALVES HSG. Práticas de empoderamento feminino na América Latina. Rev.estud.soc, 2016; 56: 80-90.

12. MEDEIROS LMOP, BATISTA SHSS. Humanização na formação e no trabalho em saúde: uma análise da literatura. Trab. educ. saúde, 2016; 14(3):925-951.

13. MEDEIROS RMK, et al. Cuidados humanizados: a inserção de enfermeiras obstétricas em um hospital de ensino. Rev. Bras. Enferm, 2016; 69(6): 1091-1098.

14. MOURA FMJSP, et al. A humanização e a assistência de enfermagem ao parto normal. Rev. bras. Enferm, 2007; 60(4): 452-455.

15. ORGANIZAÇÃO MUNDIAL DA SAÚDE - OMS. Intrapartum care for a positive childbirth experience. Geneva: World Health Organization, 2018.

16. PAGANINI AA, et al. Atuação do enfermeiro no parto humanizado. Revista Científica Eletrônica de Enfermagem, 2018; 1(1).

17. PEREIRA TCB, et al. Métodos não farmacológicos para alívio da dor no trabalho de parto: uma revisão sistemática de literatura, 2016.

18. POSSATI AB, et al. Humanização do parto: significados e percepções de enfermeiras. Esc. Anna Nery, 2017; 21(4).

19. TEIXEIRA M, et al. percepções de primíparas sobre orientações no pré natal acerca do aleitamento materno. Rev Rene, 2016.

20. TOSTES NA, SEIDL EMF. Expectativas de Gestantes sobre o Parto e suas Percepções acerca da Preparação para o Parto. Temas em Psicol, 2016; 24(2): 681-693.

21. SANCHES METL, et al. Atuação da enfermeira obstétrica na assistência ao trabalho de parto e parto. Revista Enfermagem UERJ, 2019.

22. SANTOS GO, et al. Discurso de mulheres sobre a experiência do parto normal e da cesariana. Rev Fund Care Online, 2018; 10(1):233-241.

23. SILVA AF, et al. Atuação do enfermeiro obstetra na assistência ao parto: saberes e práticas humanizadas. Brazilian Journal of Surgery and Clinical Research, 2018; 23 (3): 87-93.

24. SILVA DC, et al. Perspectiva das puérperas sobre a assistência de enfermagem humanizada no parto normal. Revista brasileira de educação e saúde, 2015; 5(2): 50-56.

25. VELHO MB, et al. Parto normal e cesárea: representações sociais de mulheres que os vivenciaram. Rev Bras Enferm, 2014; 67(2): 282-9. 